\title{
Discussion on the Effectiveness of the Copula-GARCH Method to Detect Risk of a Portfolio Containing Bitcoin
}

\author{
Ting-Yu Chen, Leh-Chyan So* \\ Department of Quantitative Finance, National Tsing Hua University, Taiwan \\ Email: *1cso@mx.nthu.edu.tw
}

How to cite this paper: Chen, T.-Y. and So, L.-C. (2020) Discussion on the Effectiveness of the Copula-GARCH Method to Detect Risk of a Portfolio Containing Bitcoin. Journal of Mathematical Finance, 10, 499-512.

https://doi.org/10.4236/jmf.2020.104030

Received: July 6, 2020

Accepted: October 7, 2020

Published: October 10, 2020

Copyright $\odot 2020$ by author(s) and Scientific Research Publishing Inc. This work is licensed under the Creative Commons Attribution International License (CC BY 4.0).

http://creativecommons.org/licenses/by/4.0/

\begin{abstract}
Since it was invented by Satoshi Nakamoto in 2008, Bitcoin has drawn considerable attention both from the financial industry and government supervisory departments, and there is no unanimity on Bitcoin's nature in the academic field. Some people may think Bitcoin is more like an asset than a currency. And investors' motivations for incorporating Bitcoin into their portfolios may vary. Might there be a better way to deal with the risk-detection issue associated with such a unique and ambiguous object? The copula-GARCH method has been proven in much of the literature to be a better way than the traditional ways to estimate the value at risk $(\mathrm{VaR})$ of portfolios. When it comes to a portfolio containing Bitcoin, can it still maintain its superiority? In this study, gold and Ethereum were each used to construct a portfolio with Bitcoin. We collected a total of 2246 daily adjusted closing prices from July 23, 2010, to March 12, 2019. As for the copula-GARCH model, we selected four constant and two time-varying copula models combined with GARCH Student- $t$ residuals to fit the joint distribution of the two assets in the portfolios. The traditional methods refer to the historical simulation, the variance-covariance method, the EWMA method, and the univariate GARCH VaR method. We adopted each method to compute corresponding one-day VaRs. Our results indicated that for the portfolios containing Bitcoin and Ethereum, the copula-GARCH method performed better than traditional methods, while for the portfolio consisting of Bitcoin and gold, traditional methods performed better. Our results may suggest that the copula-GARCH method may not be suitable in the extremely low correlation case.
\end{abstract}

\section{Keywords}

Copula, GARCH, VaR, Bitcoin, Gold, Ethereum 


\section{Introduction}

With the goal of creating an alternative to the traditional bank system, Satoshi Nakamoto invented Bitcoin in 2008. One important feature of Bitcoin is that there is no central authority to control it. Another feature is that it can be delivered on a peer-to-peer network without intermediaries. Transaction records are kept in a public system known as a blockchain. Using computer programs, miners endeavor to solve mathematical problems, and the winner can obtain one block of Bitcoin as a reward [1] ${ }^{1}$.

As its popularity rises among investors, Bitcoin has drawn considerable attention from the financial industry and supervisory departments. Some literature has also mentioned the characteristics of Bitcoin (e.g. Dyhrberg, 2016 [2]; Urquhart, 2017 [3]). From one perspective, Bitcoin is treated as an asset instead of a currency (e.g. Baek and Elbeck, 2015 [4]). Investors in the Bitcoin market are also highly speculative, resulting in its relatively high volatility. Due to its uniqueness, Bitcoin has become a hot issue in portfolio management. The role of Bitcoin in the portfolio has been analyzed in some of the literature, but there is no unanimity on the function of Bitcoin in portfolio management. For example, Eisl et al. (2015) [5] suggested that Bitcoin should be in optimal portfolios. Even though the inclusion of Bitcoin raised the conditional $\mathrm{VaR}$ of portfolios, the higher risk is covered by higher returns. Dyhrberg (2016b) [6] concluded that Bitcoin can be adapted to hedge against the FTSE index. But Bouri et al. (2016) [7] showed that Bitcoin did not perform well as a hedge against stocks, bonds, oil, etc., whereas it is suitable for diversification purposes. Bouri et al. (2017) [8] stated that before the Bitcoin price crash in December 2013, it had hedge and safe-haven characteristics against commodities; after that period, it could merely be a diversifier.

In recent years, some investors have called Bitcoin "a new form of gold". And there are some similarities between them. First, both are scarce and require great effort to extract. Bitcoin has a limited amount of about 21 million units, and gold is a well-known precious metal, since it is difficult to mine. The second similarity is their recognizability. Bitcoin has signature characteristics, so it cannot be counterfeited, and the purity of gold can be identified by doing purity tests. Third, neither is backed by a central bank or a government institution. Some literature focuses on comparisons between Bitcoin and gold. Dyhrberg (2016) [2] showed that Bitcoin is something between gold and the US dollar. However, Baur et al. (2018) [9] showed that compared to gold, Bitcoin presents obviously different returns and volatility characteristics. Klein et al. (2018) [10] found that gold played the role of a safe haven when the market dropped, while Bitcoin's trend was positively correlated with downward markets.

In the market, both Bitcoin and gold have their own advocates. People lured by Bitcoin say that it can keep its value during a financial crisis (e.g. the Bitcoin price rose when the Russian ruble crisis and the Greece debt crisis occurred),

${ }^{1}$ Refer to Nakamoto (2008) [1] for more details. 
and it provides a higher return than gold. On the other hand, gold enthusiasts say that the volatility of Bitcoin is too high, and its price is vulnerable to government regulations. If Bitcoin is banned by a government or is affected by some severe events, the capital in Bitcoin might flow into the gold market. The rise of the price of gold can compensate for the loss of Bitcoin and act as a protection. But in reality, opportunists might not treat this situation as a multiple choice; investors might have their cake and eat it too. Let us consider a hypothetical situation. How do investors deal with portfolio management when they put both Bitcoin and gold into the portfolio? In one part of this study, we discuss a portfolio constructed with Bitcoin and gold.

In addition to traditional assets like gold, we propose that other cryptocurrencies might also be incorporated with Bitcoin to form a portfolio when investors consider their asset allocation. The possible reasons are as follows. First, there are about 1500 kinds of cryptocurrencies, more than 500 of them having a scale over ten million dollars, which implies sufficient liquidity. Second, new kinds of cryptocurrency are invented every year, so there might be more choices when diversifying the portfolio. Among all cryptocurrencies, Bitcoin and Ethereum are two of the most iconic ones in the market, accounting for a $40 \%$ and $30 \%$ market share, respectively. So in another part of our study, we also design a portfolio containing Bitcoin and Ethereum.

Among several interesting issues in portfolio management, risk management is the most crucial one when it comes to cryptocurrency investments. Due to increasing speculative demand, the role of cryptocurrencies is not as purely digital money; they become a kind of investment asset. It is obvious that investors are attracted by the high return and volatility and are eager to achieve abnormal returns. However, because investors in cryptocurrencies are mainly speculators, speculative bubbles are prone to occur, and the bursting of a bubble might create great volatility shocks that affect financial markets worldwide.

When measuring risk, value at risk ( $\mathrm{VaR})$ is commonly applied to quantify the risk within a firm or portfolio over a specific period. In financial textbooks, there are some typical methods for obtaining the VaR, such as historical simulation, variance-covariance, exponentially weighted moving average, etc. One problem is that we need to know the joint distribution first when applying these methods. In practice, the joint distribution of two assets is often unknown and complex. Hence, people apply copula theory to allow the joint distribution to consist of different margins and different dependent structures.

Heteroscedasticity is an important feature in financial markets. That is, the volatilities vary with time. This feature is often fitted by the GARCH model. Another two features of financial market volatilities are leverage and asymmetry (e.g. McAleer, 2014 [11]). Previous studies applied several kinds of GARCH models to Bitcoin volatilities, such as the TGARCH (Bouri et al., 2017 [8]), the EGARCH (Bouoiyour and Selmi, 2016 [12]), etc. Hansen and Lunde (2005) [13] showed that when it came to the ability to describe conditional variance, there was no evidence that other $330 \mathrm{ARCH}$-type models performed better than the 
GARCH $(1,1)$ model. So in this study, we only applied the GARCH $(1,1)$ with Student- $t$ residuals to fit the asset returns.

The combination of GARCH and copula was frequently used when computing VaR (e.g. Lu et al., 2014 [14]; So and Yu, 2015 [15]; Huang and So, 2018 [16]). Lu et al. (2014) used static and time-varying copulas with the GARCH model to establish the conditional distribution of crude oil futures and natural gas futures. So and $\mathrm{Yu}$ (2015) [15] showed that the time-varying symmetrized Joe-Clayton (tvSJC) copula combined with the GARCH with Student- $t$ residuals performed better than traditional VaR methods. Huang and So (2018) [16] also found that when estimating the VaRs of CDS portfolios, the tvSJC copula combined with GARCH with skewed-t residuals performed the best, regardless of market situations.

The purpose of this study was to find a good way to estimate the VaR of Bitcoin portfolios. To the best of our knowledge, although there is a great deal of literature studying Bitcoin's role in a portfolio, our work is probably the first to estimate the VaR of a Bitcoin portfolio using different models. We applied the copula-GARCH method and the other four traditional VaR methods to two Bitcoin portfolios. Then we compared the results with the expected outcome to see which method performed the best.

The remainder of the paper is organized as follows. The methodology is in Section 2, the data are in Section 3, the empirical results are in Section 4, and the conclusions are in the final section.

\section{Methodology}

\subsection{Value at Risk}

$\mathrm{VaR}$ is an attempt to provide a number to summarize the risk of a portfolio. It is widely used by financial institutions. Given the time horizon ( $T$ days) and the confidence level $(1-\alpha) \cdot 100 \%$, the definition of VaR is: we are $(1-\alpha) \cdot 100 \%$ certain that the loss will not exceed $\mathrm{VaR}$ in the following $T$ days.

Among all the traditional methods, the following four are most commonly used-historical simulation, the variance-covariance method, the exponentially weighted moving average (EWMA), and the univariate GARCH-VaR method.

The univariate GARCH-VaR method applied in this study is described as follows:

Suppose $x_{p, t}(t=1,2, \cdots, T)$ are portfolio returns. We used the GARCH $(1,1)$ model with residual terms following Student- $t$ distribution to fit the portfolio return:

$$
\begin{gathered}
e_{t}=x_{p, t}-\mu, \\
\varepsilon_{t}=\frac{e_{t}}{\sqrt{h_{t}}}, \varepsilon_{t} \sim t_{v}, \\
h_{t}=\omega+\alpha \cdot e_{t-1}^{2}+\beta \cdot h_{t-1},
\end{gathered}
$$

where $\mu=E\left(x_{p, t}\right)$ is the unconditional mean and $h_{t}=\operatorname{var}\left(x_{p, t} \mid \varphi_{t-1}\right)$ is the 
conditional variance; $\varphi_{t-1}$ is the information obtained on day $t-1$. The reason we chose GARCH- $t$ was that its AIC statistic was smaller than GARCH with normal residuals. The AIC statistics of GARCH- $t$ and GARCH with skewness Student- $t$ residuals were very close, so we decided to apply the simpler one.

After fitting the portfolio returns of the last $T$ days with the GARCH $(1,1)$ model, we then estimated the conditional variance of day $T+1$. And the 1-day VaR on that day, $\operatorname{VaR}_{T+1}(\alpha)=\mu+\sqrt{h_{T+1}} \cdot \psi^{-1}(\alpha) \cdot \psi^{-1}(\alpha)$ is the inverse cumulative distribution function of Student- $t$.

\subsection{Copula-GARCH Model}

\subsubsection{Sklar's Theorem}

A copula is a multidimensional probability distribution function with uniform-distributed marginal distributions. Given $n$ random variables $\left\{x_{1}, x_{2}, \cdots, x_{n}\right\}$ and $F$ denoting an $n$-dimensional distribution function with marginal distribution $F_{1}, F_{2}, \cdots, F_{n}$, then there exists a copula representation such that:

$$
\begin{aligned}
F\left(x_{1}, x_{2}, \cdots, x_{n}\right) & =P\left(X_{1} \leq x_{1}, \cdots, X_{n} \leq x_{n}\right) \\
& =C\left(P\left(X_{1} \leq x_{1}\right), \cdots, P\left(X_{n} \leq x_{n}\right)\right) \\
& =C\left(F_{1}\left(x_{1}\right), \cdots, F_{n}\left(x_{n}\right)\right)
\end{aligned}
$$

If all variables are continuous, $u_{i}=F_{i}\left(x_{i}\right)$ follows the uniform distribution, and $f_{i}$ denotes the marginal probability distribution functions. According to Sklar's theorem, any multivariate probability distribution function is composed of a copula and marginal probability distribution functions.

$$
\begin{aligned}
f\left(x_{1}, \cdots, x_{n}\right) & =\frac{\partial F\left(x_{1}, \cdots, x_{n}\right)}{\partial x_{1} \cdots \partial x_{n}} \\
& =\frac{\partial C\left(u_{1}, \cdots, u_{n}\right)}{\partial u_{1} \cdots \partial u_{n}} \cdot \prod_{i} \frac{\partial F_{i}\left(x_{i}\right)}{\partial x_{i}} \\
& =c\left(u_{1}, \cdots, u_{n}\right) \cdot \prod_{i} f_{i}\left(x_{i}\right)
\end{aligned}
$$

From the equations above, the marginal distributions need not be the same as each other, nor does the selection of the copula need to be the same as the choice of marginal distributions. The following are some copulas that are commonly used.

\subsubsection{Copula Functions}

1) Normal copula

$$
C_{\text {Normal }}\left(u_{1}, u_{2}, \rho\right)=\Phi_{\rho}\left[\Phi^{-1}\left(u_{1}\right), \Phi^{-1}\left(u_{2}\right)\right],
$$

where $\Phi_{\rho}$ is the bivariate standard normal cumulative distribution function with correlation coefficient $\rho$.

2) Student- $t$ copula

$$
C_{T}\left(u_{1}, u_{2}, \rho, v\right)=t_{\rho, v}\left[t_{v}^{-1}\left(u_{1}\right), t_{v}^{-1}\left(u_{2}\right)\right],-1 \leq \rho \leq 1
$$

where $t_{\rho, v}$ is the bivariate Student- $t$ cumulative distribution function, $\rho$ is 
the correlation, and $v$ is the degree of freedom. When the degree of freedom is large enough, the Student- $t$ copula converges to a Gaussian copula. It is often applied to capture the fat-tailed dependence feature of financial data.

3) Clayton copula

$$
C_{\text {Clayton }}\left(u_{1}, u_{2} ; \omega\right)=\left(u_{1}^{-\omega}+u_{2}^{-\omega}-1\right)^{\frac{-1}{\omega}}, \omega \in[-1, \infty) \backslash\{0\} .
$$

The Clayton (1978) [17] copula is asymmetric. When $u_{1}$ or $u_{2}$ approach 0 , the value of the copula goes up, meaning that the dependence of these two variables becomes heavier. Thus, it is suitable for capturing the dependence around the left tail.

4) Symmetrized Joe-Clayton copula (SJC copula)

The Joe-Clayton copula (JC copula) has the following form:

$$
\begin{gathered}
C_{J C}\left(u_{1}, u_{2} \mid \tau^{U}, \tau^{L}\right)=1-\left(1-\left\{\left[1-\left(1-u_{1}\right)^{k}\right]^{-\gamma}+\left[1-\left(1-u_{2}\right)^{k}\right]^{-\gamma}-1\right\}^{\frac{-1}{\gamma}}\right)^{\frac{1}{k}}, \\
k=\frac{1}{\log _{2}\left(2-\tau^{U}\right)}, \gamma=\frac{-1}{\log _{2}\left(2-\tau^{L}\right)}, \tau^{U} \in(0,1), \tau^{L} \in(0,1), \\
\tau^{U}=\lim _{\varepsilon \rightarrow 0} \operatorname{Pr}\left(x_{2}>F_{2}^{-1}(\varepsilon) \mid x_{1}>F_{1}^{-1}(\varepsilon)\right), \\
\tau^{L}=\lim _{\varepsilon \rightarrow 0} \operatorname{Pr}\left(x_{2} \leq F_{2}^{-1}(\varepsilon) \mid x_{1} \leq F_{1}^{-1}(\varepsilon)\right),
\end{gathered}
$$

where $\tau^{U}$ is the upper tail dependence, and $\tau^{L}$ is the lower tail dependence. From the two equations above, $\tau^{U}$ is the probability that $x_{2}$ has an extremely large increase, given that $x_{1}$ has an extremely large increase. On the other hand, $\tau^{L}$ measures the probability that $x_{2}$ has an extremely large decline, given that $x_{1}$ has an extremely great decline.

The SJC copula is a modified version of the JC copula:

$$
\begin{aligned}
& C_{S J C}\left(u_{1}, u_{2} \mid \tau^{U}, \tau^{L}\right) \\
& =0.5 \cdot\left(C_{J C}\left(u_{1}, u_{2} \mid \tau^{U}, \tau^{L}\right)+C_{J C}\left(1-u_{1}, 1-u_{2} \mid \tau^{U}, \tau^{L}\right)+u_{1}+u_{2}-1\right) .
\end{aligned}
$$

5) Time-varying normal copula

The copulas mentioned above are all static. That is, the dependence structure between two assets remains the same over time. However, the real situation is that dependence structure varies across time. Hence, the time-varying copula is proposed. According to Patton (2006), the time-varying copula has the following form:

$$
\begin{gathered}
C_{t v \text { Normal }}\left(u_{1}, u_{2} ; \rho_{t}\right)=\Phi\left[\Phi^{-1}\left(u_{1}\right), \Phi^{-1}\left(u_{2}\right)\right], \\
\rho_{t}=\tilde{\Lambda}\left(\omega_{\rho}+\beta_{\rho} \cdot \rho_{t-1}+\alpha_{\rho} \cdot 0.1 \sum_{j=1}^{10} \Phi^{-1}\left(u_{1, t-j}\right) \cdot \Phi^{-1}\left(u_{2, t-j}\right)\right),
\end{gathered}
$$

where $\tilde{\Lambda}(x)=\tanh \left(\frac{x}{2}\right)$, which is a transformation to let $\rho_{t}$ stay in the range of -1 and 1 . 
6) Time-varying symmetrized Joe-Clayton copula (tvSJC copula)

According to Patton (2006) [18], the tvSJC copula has the same function form as the SJC copula. The difference is that its dependence parameters follow a specific form of evolution.

$$
\begin{gathered}
C_{t v S J C}\left(u_{1}, u_{2} \mid \tau_{t}^{U}, \tau_{t}^{L}\right) \\
=0.5 \cdot\left(C_{J C}\left(u_{1}, u_{2} \mid \tau_{t}^{U}, \tau_{t}^{L}\right)+C_{J C}\left(1-u_{1}, 1-u_{2} \mid \tau_{t}^{U}, \tau_{t}^{L}\right)+u_{1}+u_{2}-1\right) \\
\tau_{t}^{U}=\Lambda\left(\omega_{U}+\beta_{U} \cdot \tau_{t-1}^{U}+\alpha_{U} \cdot \frac{1}{10} \sum_{j=1}^{10}\left|u_{1, t-j}-u_{2, t-j}\right|\right), \\
\tau_{t}^{L}=\Lambda\left(\omega_{L}+\beta_{L} \cdot \tau_{t-1}^{L}+\alpha_{L} \cdot \frac{1}{10} \sum_{j=1}^{10}\left|u_{1, t-j}-u_{2, t-j}\right|\right), \\
\Lambda=\left(1-\mathrm{e}^{-x}\right)^{-1}, \tau_{t}^{U} \in(0,1), \tau_{t}^{L} \in(0,1) .
\end{gathered}
$$

$\Lambda$ is a logistic transformation to make $\tau_{t}^{U}$ and $\tau_{t}^{L}$ stay in the range of 0,1, $\tau_{t}^{U}$ is the upper tail dependence, and $\tau_{t}^{L}$ is the lower tail dependence.

\subsubsection{Parameter Estimation}

Assume there are two random variables $x_{1}$ and $x_{2}$, and each has $T$ observations $\left\{\left(x_{1 t}, x_{2 t}\right)\right\}_{t=1}^{T}$. The joint cumulative distribution function and probability distribution function are:

$$
\begin{gathered}
F\left(x_{1}, x_{2}\right)=C\left[F_{1}\left(x_{1 t}, \theta_{1}\right), F_{2}\left(x_{2 t}, \theta_{2}\right) ; \theta\right] \\
f\left(x_{1}, x_{2}\right)=c\left[F_{1}\left(x_{1 t}, \theta_{1}\right), F_{2}\left(x_{2 t}, \theta_{2}\right) ; \theta\right] \cdot f_{1}\left(x_{1 t}, \theta_{1}\right) \cdot f_{2}\left(x_{2 t}, \theta_{2}\right),
\end{gathered}
$$

where $\theta_{1}$ and $\theta_{2}$ are the GARCH parameters, and $\theta$ is the copula parameter. Let $\varphi=\left(\theta_{1}, \theta_{2}, \theta\right)$. In this case, the log-likelihood function is:

$$
\begin{gathered}
\ln L(\varphi)=\sum_{t=1}^{T} \ln \left\{c\left[F_{1}\left(x_{1 t}, \theta_{1}\right), F_{2}\left(x_{2 t}, \theta_{2}\right) ; \theta\right]\right\}+\sum_{t=1}^{T} \sum_{i=1}^{2} \ln \left[f_{i}\left(x_{i t}, \theta_{i}\right)\right] \\
\hat{\varphi}_{M L E}=\arg \max \ln L(\varphi) .
\end{gathered}
$$

If the GARCH parameters and the copula parameters are estimated simultaneously, the computation will take too much time. Hence, Joe (2005) [19] proposed the two-stage inference function for margins (IFMs) method to estimate the parameters separately.

In the first step, we only consider the log-likelihood function of the marginal distributions and estimate the GARCH parameters:

$$
\begin{gathered}
\ln L_{i}\left(\theta_{i}\right)=\sum_{t=1}^{T} \ln \left[f_{i}\left(x_{i t}, \theta_{i}\right)\right], i=1,2 \\
\hat{\theta}_{i_{M L E}}=\arg \max \ln L_{i}\left(\theta_{i}\right), \quad i=1,2
\end{gathered}
$$

In the second step, we substitute the parameters estimated in stage 1 into the following equation and apply MLE to estimate the copula parameter:

$$
\ln L(\theta)=\sum_{t=1}^{T} \ln \left\{c\left[F_{1}\left(x_{1 t}, \hat{\theta}_{1}\right), F_{2}\left(x_{2 t}, \hat{\theta}_{2}\right) ; \theta\right]\right\}
$$




$$
\hat{\theta}_{M L E}=\arg \max \ln L(\theta) .
$$

\subsubsection{VaR Using Copula-GARCH}

Suppose we have the data of two asset returns $\left\{\left(r_{1 t}, r_{2 t}\right)\right\}_{t=1}^{T}$, which is the in-sample data. We want to estimate their joint distribution on day $T+1$ and the $\mathrm{VaR}$ on day $T+1$. The procedures are as follows:

1) Use the GARCH model to fit $\left(r_{1 t}\right)_{t=1}^{T}$ and $\left(r_{2 t}\right)_{t=1}^{T}$. Then obtain the standardized residuals.

2) Apply probability integral transform to the standardized residuals to make them uniformly distributed. After the transformation, we obtain $\left(u_{1 t}\right)_{t=1}^{T}$ and $\left(u_{2 t}\right)_{t=1}^{T}$.

3) Estimate the copula parameters by applying the maximum likelihood method.

4) Substitute the estimated parameters into the copula to obtain a uniformly distributed random vector $\left\{\left(v_{1 t}, v_{2 t}\right)\right\}_{t=1}^{T}$.

5) Apply probability integral transform to $\left\{\left(v_{1 t}, v_{2 t}\right)\right\}_{t=1}^{T}$ and substitute the unconditional mean and conditional variance of the GARCH models to obtain a set of simulated asset returns $\left\{\left(w_{1 t}, w_{2 t}\right)\right\}_{t=1}^{T}$.

6) Suppose two assets are equally weighted in the portfolio. Use the results of step (5) to obtain the simulated portfolio returns and then reorder them. The $[T \times \alpha]^{\text {th }}$ worst value on the reordered list is $\mathrm{VaR}_{T+1}$. Assume $\alpha$ is $5 \%$ or $1 \%$.

7) Suppose there are $N$ out-of-sample data. When a new observation is added, the oldest one is removed. Repeat step (1) to step (6) $N$ times to obtain all out-of-sample 1-day VaRs.

\subsubsection{Back Testing}

We compared the actual portfolio return to the corresponding 1-day VaR. If the confidence level was $(1-\alpha) \cdot 100 \%$, we expected $N \times \alpha$ actual portfolio returns to be lower than the 1-day VaR. The method giving the closet number of violations to the expected number of violations performs the best.

Suppose there are $N$ out-of-sample observations and $y$ violations. Let $p=y / N$. The hypothesis testing is $H_{0}: p^{*}=\alpha$ vs. $H_{1}: p^{*} \neq \alpha$. Here we applied the likelihood ratio test (Kupiec, 1995 [20]), and the test statistic is:

$$
\mathrm{LR}=-2 \cdot \ln \left[\alpha^{y}(1-\alpha)^{N-y}\right]+2 \cdot \ln \left[\left(\frac{y}{N}\right)^{y}\left(1-\frac{y}{N}\right)^{N-y}\right] \sim x^{2}(1) \text { under } H_{0}
$$

Using the information above, we obtained the non-rejection region of $H_{0}$. For example, when $N=600$ and $\alpha=0.05$, the non-rejection region of $H_{0}$ is $21 \leq y \leq 41$.

If the number of violations lies within the non-rejection region, it means the method applied to calculate $\mathrm{VaR}$ is appropriate. In contrast, if it lies outside the non-rejection region, it means the method overestimates or underestimates the risk. 


\section{Data}

The daily log returns of each asset are computed as follows:

$$
r_{t}=\ln \left(\frac{P_{t}}{P_{t-1}}\right),
$$

where $P_{t}$ and $P_{t-1}$ are the adjusted closing prices of each asset. In this paper, we use both gold and Ethereum to form portfolios with Bitcoin.

\subsection{Portfolio Containing Bitcoin and Gold}

Both Bitcoin and gold data were obtained from the Bloomberg database; in this database, the first report of Bitcoin price data was July 23, 2010. Hence, we set the starting date of the portfolio on that day. We collected a total of 2246 daily adjusted closing prices from July 23, 2010, to March 12, 2019, and obtained 2245 daily log returns. The first 1000 returns from July 26, 2010, to June 2, 2014, are used as in-sample data, and the remaining 1245 returns from June 3, 2014, to March 12, 2019, are used as out-of-sample data.

\subsection{Portfolio Containing Bitcoin and Ethereum}

The Bitcoin data was collected from the Bloomberg database. The Ethereum data was not available on the Bloomberg database, so we obtained it from the Coindesk website. The start date of Ethereum price data on this website is August 7, 2015. We collected a total of 938 daily adjusted closing prices ranging from August 7, 2015, to March 12, 2019, and obtained 937 daily log returns. The first 600 returns from August 10, 2015, to November 24, 2017, are used as in-sample data, and the remaining 337 returns from November 27, 2017, to March 12, 2019, are used as out-of-sample data.

\section{Empirical Results}

\subsection{Copulas}

In this section, we combined the GARCH- $t$ model with six kinds of copulas, including four static copulas and two dynamic copulas, to model the joint distribution of two asset returns. The estimated copula parameters are shown in $\mathrm{Ta}$ ble 1. We used the AIC statistics to determine which copula fitted the best. The results are shown in Table 2. For the portfolio containing Bitcoin and gold, the time-varying normal copula with GARCH- $t$ had the lowest AIC, and for the portfolio containing Bitcoin and Ethereum, the $t$-copula with GARCH- $t$ had the best goodness-of-fit. One thing worth mentioning is that for these two portfolios, the dynamic copula-GARCH models did not necessarily perform better than the static ones. According to previous literature (e.g. So and Yu, 2015 [15]), the dynamic ones have the characteristic of capturing time-varying relations between two asset returns, so they usually perform better than the static ones. But in this case, maybe because of low correlation between asset returns, the dynamic ones may not perform better than the static ones. 
Table 1. Results of copula-GARCH- $t$ models.

\begin{tabular}{cccc}
\hline Copula & Parameter & Bitcoin \& Gold & Bitcoin \& Ethereum \\
\hline Normal & $\rho$ & -0.056144 & 0.066045 \\
Student $t$ & $\rho$ & -0.056434 & 0.052308 \\
Clayton & $V$ & 99.985635 & 7.891662 \\
SJC & $\delta$ & 0.000103 & 0.094013 \\
& $\lambda_{u}$ & $6.019420 \times 10^{-7}$ & $6.267916 \times 10^{-5}$ \\
tvNormal & $\lambda_{L}$ & $7.379685 \times 10^{-7}$ & 0.012719 \\
& $\omega_{\rho}$ & -0.038916 & 0.298822 \\
& $\alpha_{\rho}$ & -0.069228 & -0.392363 \\
& $\beta_{\rho}$ & 1.402669 & -1.910929 \\
& $\alpha_{u}$ & -15.301512 & -15.203831 \\
& $\beta_{u}$ & -0.379823 & 1.938233 \\
tvSJC & $\omega_{u}$ & -0.000174 & -0.010136 \\
& $\alpha_{L}$ & -15.329840 & 2.446052 \\
& $\beta_{L}$ & -0.475029 & -24.999134 \\
& $\omega_{L}$ & -0.005585 & -0.866843 \\
\hline & & &
\end{tabular}

After fitting in-sample returns of each portfolio with the GARCH- $t$ model, we applied probability integral transform to the standardized residuals to get uniformly distributed vectors. Then we put them into different kinds of copulas. This table shows the estimated parameters of six copulas for the two portfolios.

Table 2. AIC of copula-GARCH models.

\begin{tabular}{ccc}
\hline Copula & Bitcoin \& Gold & Bitcoin \& Ethereum \\
\hline Normal & -3.155078 & -2.619534 \\
Student- $t$ & -2.644454 & -9.959406 \\
Clayton & 0.011215 & -4.623443 \\
SJC & 6.120127 & -5.574239 \\
tv normal & -4.005762 & -5.038872 \\
tv SJC & 15.310120 & -6.549664 \\
\hline
\end{tabular}

We chose the copula-GARCH model by AIC statistics. The lowest AIC statistic among all is marked in bold.

\subsection{Backtesting of VaR Using Five Methods}

The five methods applied to the estimation of $\mathrm{VaR}$ were historical simulation, the variance-covariance method, EWMA, the univariate GARCH method, and the copula-GARCH method. We set the first T days as the in-sample period and tried to estimate the 1-day $\mathrm{VaR}_{T+1}$. In the portfolio containing Bitcoin and gold, there were 1000 in-sample data and 1245 out-of-sample data; for the portfolio containing Bitcoin and Ethereum, there were 600 in-sample data and 337 out-of-sample data. We applied the rolling window method. For example, we used the data from day 2 to day 1001 to estimate $\mathrm{VaR}_{1002}$; day 3 to day 1002 to estimate $\mathrm{VaR}_{1003}$, etc. 
For the portfolio containing Bitcoin and gold, historical simulation and EWMA passed backtesting under a 95\% confidence level. If the confidence level was $99 \%$, variance-covariance and GARCH- $t$ were the only two that passed backtesting. One thing worth mentioning is that in this portfolio, whether the confidence level was $95 \%$ or $99 \%$, the time-varying normal copula-GARCH- $t$ model did not perform well. It gave out lower numbers of violations, which means it overestimated the risk of the portfolio. In some previous literature (e.g. So and Yu, 2015 [15]; Huang and So, 2018 [16]), the copula-GARCH model performed much better than traditional methods, which differs from the results here. Some literature (e.g. Eisl et al., 2015 [5]; Chuen et al., 2017 [21]) showed that Bitcoin returns had extremely low correlations with traditional asset returns such as currencies, stocks, bonds, gold, etc. The correlation coefficient between Bitcoin returns and gold returns is about -0.004 , which is extremely low. Hence, the copula model may not be suitable in this case. Instead, the traditional methods perform better than the copula-GARCH model. Among the four traditional methods, when $\alpha$ is $5 \%$, historical simulation is the most accurate among them all; when $\alpha$ is $1 \%$, the variance-covariance method gives out the closet violation numbers. Hence, we speculated that when correlation is low, the performance of traditional methods may vary case by case.

As for the portfolio containing Bitcoin and Etheruem, historical simulation and the $t$-copula-GARCH- $t$ model passed backtesting under $95 \%$ and $99 \%$ confidence levels. The variance-covariance method only passed backtesting under a $95 \%$ confidence level, and GARCH- $t$ only passed backtesting under a $99 \%$ confidence level. Among all the methods, $t$-copula-GARCH- $t$ gave out the number of violations closest to the expected number under both $95 \%$ and $99 \%$ confidence levels.

When we compared the two portfolios, the difference was the correlation coefficient between asset returns. In the former, the correlation coefficient was only -0.004; while in the latter, it was about 0.2 . Although 0.2 is not a very high correlation coefficient compared to previous literature (e.g. Huang and So, 2018), the copula-GARCH model still showed an advantage in this case.

The backtesting results of different VaR models are presented in Table 3. The EWMA and univariate GARCH methods gave out a relatively high number of violations, which means an underestimation of risk. The copula-GARCH method considers both serial correlations and the nonlinear dependence of asset returns. Theoretically, it is the best method of estimating VaR. But when the correlation is too low, as in the case of the portfolio consisting of Bitcoin and gold, the result might be contrary to the expected outcome.

\section{Conclusions}

In recent years, the trading volume and popularity of Bitcoin have risen significantly, providing another choice for investors worldwide to make their portfolios more diverse. To deal with the drastic trend of the Bitcoin price, we endeavored to find an effective way to estimate the VaR of Bitcoin portfolios. 
Table 3. Number of violations of the two portfolios.

\begin{tabular}{|c|c|c|}
\hline \multicolumn{3}{|c|}{ Portfolio containing Bitcoin and Gold } \\
\hline Trading days: 1245 & $\alpha=0.05$ & $\alpha=0.01$ \\
\hline Expected No. of violations & 62 & 12 \\
\hline Non-rejection regions & $48 \leq y \leq 75$ & $7 \leq y \leq 22$ \\
\hline Historical simulation & $60^{*}$ & 6 \\
\hline Variance-covariance & 43 & $15^{\star}$ \\
\hline EWMA & $53^{*}$ & 24 \\
\hline GARCH- $t$ & 44 & $7^{*}$ \\
\hline tvNormal-copula-GARCH- $t$ & 21 & 2 \\
\hline \multicolumn{3}{|c|}{ Portfolio containing Bitcoin and Ethereum } \\
\hline Trading days: 337 & $\alpha=0.05$ & $\alpha=0.01$ \\
\hline Expected No. of violations & 17 & 3 \\
\hline Non-rejection regions & $10 \leq y \leq 25$ & $1 \leq y \leq 9$ \\
\hline Historical simulation & $24^{*}$ & $7^{*}$ \\
\hline Variance-covariance & $25^{*}$ & 12 \\
\hline EWMA & 39 & 19 \\
\hline GARCH- $t$ & 28 & $7^{\star}$ \\
\hline$t$-copula-GARCH- $t$ & $16^{*}$ & $3^{*}$ \\
\hline
\end{tabular}

Violation numbers of all five models are presented here. The one with the closet violation number to the expected violations is the best. If the violation number lay in the non-rejection region, we marked it with a star.

One of our main contributions is that our work is probably the first to estimate the VaR of a Bitcoin portfolio using different models. According to the evidence provided by financial data, the assumption of linear correlation is improper for depicting the dependence structure. Hence, a flexible multivariate distribution with different margins and dependent structures is required. This allows the joint distribution to be free of normality and linear correlation. Hence, in addition to traditional VaR methods, we also applied the copula-GARCH model with Student- $t$ residuals to the VaR estimation.

The other main contribution of this study is that we propose a reminder for the limitation of applying the copula-GARCH method. To our surprise, even though the copula-GARCH method has been proven in much of the literature to be a better way than the traditional ways to estimate the VaR of portfolios (e.g. Lu et al., 2014 [13]; So and Yu, 2015 [14]; Huang and So, 2018 [15]), its effectiveness may not hold when it comes to a portfolio containing Bitcoin. In this study, we formed two Bitcoin portfolios, one with gold and the other with Ethereum. The results showed that if the correlation between asset returns is not extremely low, for example in the portfolio containing Bitcoin and Ethereum in this paper, the copula-GARCH method is still better than other traditional me- 
thods. However, if the correlation between two asset returns is extremely low, for example in the portfolio containing Bitcoin and gold in this paper, then the copula-GARCH model may not perform better than the other methods. Originally, the advantage of the copula-GARCH method was that it considers heteroscedasticity and allows nonlinear or more complicated dependence structures. But when the correlation is low, despite these advantages, it may be dominated by other models.

\section{Conflicts of Interest}

The authors declare no conflicts of interest regarding the publication of this paper.

\section{References}

[1] Nakamoto, S. (2008) Bitcoin: A Peer-to-Peer Electronic Cash System.

[2] Dyhrberg, A.H. (2016) Bitcoin, Gold and the Dollar-A GARCH Volatility Analysis. Finance Research Letters, 16, 85-92. https://doi.org/10.1016/j.frl.2015.10.008

[3] Urquhart, A. (2017) Price clustering in Bitcoin. Economics Letters, 159, 145-148. https://doi.org/10.1016/j.econlet.2017.07.035

[4] Baek, C. and Elbeck, M. (2015) Bitcoins as an Investment or Speculative Vehicle? A First Look. Applied Economics Letters, 22, 30-34. https://doi.org/10.1080/13504851.2014.916379

[5] Eisl, A., Gasser, S. and Weinmayer, K. (2015) Caveat Emptor: Does Bitcoin Improve Portfolio Diversification?

[6] Dyhrberg, A.H. (2016) Hedging Capabilities of Bitcoin. Is It the Virtual Gold? Finance Research Letters, 16, 139-144. https://doi.org/10.1016/j.frl.2015.10.025

[7] Bouri, E., Molnár, P., Azzi, G., Roubaud, D. and Hagfors, L. (2016) On the Hedge and Safe Haven Properties of Bitcoin: Is It Really More than a Diversifier? Finance Research Letters, 20, 192-198. https://doi.org/10.1016/j.frl.2016.09.025

[8] Bouri, E., Jalkh, N.P., Molnár, P. and Roubaud, D. (2017) Bitcoin for Energy Commodities before and after the December 2013 Crash: Diversifier, Hedge or Safe Haven? Applied Economics, 49, 5063-5073. https://doi.org/10.1080/00036846.2017.1299102

[9] Baur, D., Dimpfl, T. and Kuck, K. (2018) Bitcoin, Gold and the US Dollar-A Replication and Extension. Finance Research Letters, 25, 103-110. https://doi.org/10.1016/j.frl.2017.10.012

[10] Klein, T., Thu, H.P. and Walther, T. (2018) Bitcoin Is Not the New Gold-A Comparison of Volatility, Correlation, and Portfolio Performance. International Review of Financial Analysis, 59, 105-116. https://doi.org/10.1016/j.irfa.2018.07.010

[11] McAleer, M. (2014) Asymmetry and Leverage in Conditional Volatility Models. Econometrics, 2, 145-150. https://doi.org/10.3390/econometrics2030145

[12] Bouoiyour, J. and Selmi, R. (2016) Bitcoin: A Beginning of a New Phase. Economics Bulletin, 36, 1430-1440.

[13] Hansen, P.R. and Lunde, A. (2005) A Forecast Comparison of Volatility Models: Does Anything Beat a GARCH $(1,1)$ ? Journal of Applied Econometrics, 20, 873-889. https://doi.org/10.1002/jae.800

[14] Lu, X.F., Lai, K.K. and Liang, L. (2014) Portfolio Value-at-Risk Estimation in Ener- 
gy Futures Markets with Time-Varying Copula-GARCH Model. Annals of Operations Research, 219, 333-357. https://doi.org/10.1007/s10479-011-0900-9

[15] So, L.C. and Yu, J.Y. (2015) Improved Detection of Rare-Event Risk of a Portfolio with U.S. REITs. Annals of Financial Economics, 10, Article ID: 1550015. https://doi.org/10.1142/S2010495215500153

[16] Huang, J.J. and So, L.C. (2018) Application of Copula-GARCH to Estimate VaR of a Portfolio with Credit Default Swaps. Journal of Mathematical Finance, 8, 382-407. https://doi.org/10.4236/jmf.2018.82025

[17] Clayton, D.G. (1978) A Model for Association in Bivariate Life Tables and Its Application in Epidemiological Studies of Familial Tendency in Chronic Disease Incidence. Biometrika, 65, 141-151. https://doi.org/10.1093/biomet/65.1.141

[18] Patton, A.J. (2006) Modelling Asymmetric Exchange Rate Dependence. International Economic Review, 47, 527-556.

[19] Joe, H. (2005) Asymptotic Efficiency of the Two-Stage Estimation Method for Copula-Based Models. Journal of Multivariate Analysis, 94, 401-419.

https://doi.org/10.1016/j.jmva.2004.06.003

[20] Kupiec, P.H. (1995) Techniques for Verifying the Accuracy of Risk Measurement Models. The Journal of Derivatives, 3, 73-84.

https://doi.org/10.3905/jod.1995.407942

[21] Chuen, K.D.L., Guo, L. and Wang, Y. (2017) Cryptocurrency: A New Investment Opportunity? The Journal of Alternative Investments Winter, 20, 16-40. 